\title{
An Energy Efficient Multihop Hierarchical Routing in Wireless sensor Networks
}

\author{
V.Chandrasekaran ${ }^{1}$, Dr.A.Shanmugam ${ }^{2}$ \\ ${ }^{I}$ (Research Scholar, Assistant Professor (Senior Grade), Department Of ECE, Velalar College of Engineering \\ and Technology,Erode, Tamil Nadu, India) \\ ${ }_{2}^{2}$ (Principal and Research Supervisor, Bannariamman Institute of Technology Sathyamangalam, Tamil Nadu, \\ India)
}

\begin{abstract}
Wireless sensor networking is an emerging technology that promises a wide range of potential applications in both civilian and military areas. The most important issue faced in all wireless sensor networks is its energy consumption. A cluster-based routing method is more energy-efficient than a flat routing method as it can only send specific data for user requirements and aggregate similar data by dividing a network into a local cluster. However, previous clustering algorithms have some problems in that the transmission radius of sensor nodes is not realistic and multi-hop based communication is not used both inside and outside local clusters. Traditional routing protocols have greatly lost its importance. In this paper we proposes an Energy Efficient Multihop Hierarchical Routing named E2MHR. It is a multi-hop routing protocol to reduce the energy consumption and increase the network life time in all sensor nodes.
\end{abstract}

Keywords - Wireless Sensor Networks, Multihop routing, Energy Efficiency.

\section{INTRODUCTION}

Wireless Sensor network is of great importance as we need to monitor and collect data from various environments. We in general use thousands and thousands of sensor nodes to sense the environment change and to exchange information between nodes and to sink. In order to many these sensor nodes cheap and small, we usually limit the battery power, processor, memory, and RF module and sensing devices. The most important issue faced in wireless sensor network is its power consumption. Energy consumption is directly associated with $\mathrm{RF}$ module which in turn is associated with communicating messages. So by limiting the RF module, energy consumption can be minimized.

Sensors having data to transmit should relay this data to a single source using multihop. Nodes that do not have data to transmit or that are not relaying the data of other nodes can be put to sleep. Energy efficiency is achieved by reducing the number of active nodes. For each node, the energies consumed during reception, transmission, and sensing are considered in the analysis.

If the communication fails between the source and a frame node, or between two frame nodes, assistant nodes come into play and relay the data to the next frame node. Hence the use of opportunistic transmissions depending on the fading conditions of the channel. The optimal number of nodes that should be included in a path is determined. The purpose is to reduce the energy consumption by reducing the number of nodes relaying the data from source to destination. In the scenario investigated in this Chapter, all nodes are assumed to have data to transmit, and hence cannot be put to sleep to achieve energy savings. This scenario corresponds, for example, to WSNs deployed for the purpose of air quality monitoring in a given area, where each sensor will periodically send measurement data to a central processing system.

Scalability is one of the important issues associated with Wireless sensor network. Depending upon the application these networks are widely used in many applications with different scales. So every algorithm should be in charge for all scale. In general routing protocol of Sensor network is categorized into 3 types: Hierarchical protocol, Flat protocol and routing protocol. The main idea behind the hierarchical protocol is to increase the network lifetime and to reduce the energy consumption.

Similar to the hierarchical routing protocol, the proposed protocol will use random number based distributed algorithm. When the sink sends the place advertisement message to the node, the node will diagnose the distance by calculating the power received. In the proposed protocol, the distance between node, limitation parameter and overhead concept is applied to form a cluster that will highly reduce the energy consumption by node. Also it uses Energy Index to send the cluster messages between nodes and to increase the network lifetime by using cluster head concept.

\section{RELATED WORK}

In this section, an overview of related work is provided, including clustering algorithms, multi-hop data transmission, and energy hole issues. Heinzelman(2000), et al. presented the LEACH (Low Energy Adaptive 
Clustering Hierarchy) protocol for WSNs of cluster-based architecture, which is a widely known and elegant clustering algorithm, by selecting the $\mathrm{CHs}$ in rounds. $\mathrm{LEACH}$ is one of the most popular hierarchical routing algorithms for sensor networks. Now the motive is to form sensor nodes clusters based on incoming signal strength and then local cluster heads are used as routers to the sink. As transmissions are done by those clusters alone, instead of sensor nodes, energy will be saved here. Given that in LEACH data collection is performed periodically, this protocol is supposed to be more suitable when there is a need for constant proactive monitoring by the WSN. However, in several cases, periodic data transmissions are needless, thus causing ineffectual expenditure of energy. LEACH achieved improvement compared to direct transmissions, as measured in terms of nodes' lifetime.

Following the descendant of LEACH is PEGASIS algorithm, which was introduced by Lindsey and Raghavendra(2002). Power-efficient Gathering in Sensor Information Systems (PEGASIS) is an improvement of the LEACH protocol. Rather than forming multiple clusters, PEGASIS forms chains from sensor nodes so that each node transmits and receives from a neighbor and only one node is selected from that chain to transmit to the base station (sink). Collected data is moved from one node to another node, then aggregated and is sent to base station. Although, PEGASIS achieves higher energy conservation when compared to LEACH, it suffers however from certain drawbacks. One of them is the excessive delay which is caused by distant nodes on the chain. Another weakness of the protocol has to do with the fact that the single leader can become a bottleneck for the network.

Hierarchical-PEGASIS, introduced by Lindsey et al, is an extension to PEGASIS. It achieves a notable decrease of the delay incurred for packets during transmission simultaneous transmissions of data. However, both PEGASIS and Hierarchical-PEGASIS, like LEACH, are based on some unrealistic assumptions such as that all nodes have the same level of energy and are able to communicate with the BS directly.

TEEN is another widely referenced and extensively used energy efficient hierarchical routing protocol, proposed by Manjeshwar and Agrawal(2001). TEEN pursues a hierarchical approach and it uses data-centric mechanism. The sensor network architecture is mainly based on a hierarchical grouping in which closer nodes will form clusters. Each cluster head collects and aggregates all data it receives from its cluster nodes and forwards the aggregated data either directly or indirectly to the base station.

Many other hierarchical routing protocols have been proposed either in the past or more recently. Similarly, many alternative approaches have been proposed for energy conservation in WSNs, for instance through optimized clustering, or collaborative sensing and adaptive target estimation.

\section{NETWORK MODEL}

We consider the network model of hundreds of sensor nodes, and be distributed in a large area. Since the sensor nodes which are far away from the BS could not communicate with the BS directly in the transmission range, all sensor nodes could reach the BS using multi-hop communications.

The radio model that is used is the same adopted in [4]. By using this approach, we assumed the energy loss of $\mathrm{d} 2$ due to channel transmission. The node dissipates the energy for the radio transmission of a message of $\mathrm{k}$ bits over a distance $\mathrm{d}$ is due to running both the transmitter circuitry and the transmitter amplifier is given by

$$
\operatorname{ETx}(\mathrm{k}, \mathrm{d})=\text { Eelec } * \mathrm{k}+\text { Eamp } * \mathrm{k} * \mathrm{~d} 2
$$

where Eelec is the transmitter circuitry dissipation per bit, which is supposed to be equal to the corresponding receiver circuitry dissipation per bit and Eamp is the transmit amplifier dissipation per bit per square meter. Similarly, the energy $\operatorname{ERx}(\mathrm{k})$ dissipated by a node for the reception of a k-bit message is due to running the receiver circuitry is given by

$$
\operatorname{ERx}(\mathrm{k})=\text { Eelec } * \mathrm{k}
$$

\section{ENERGY EFFICIENT MULTIHOP HIERARCHICAL ROUTING}

In this paper, we proposes the multihop hierarchical reactive routing algorithm named E2MHR(Energy Efficient Multihop Hierarchical Routing), which aims at the achieve of energy efficiency through the use of both multihop routing and completely distributed based on random numbers.

Proposed method makes use of distributive algorithm based on random number. The protocol uses the Energy Index for sending intra-cluster messages and selecting candidate node. Energy-Index can be obtained from the equation

$$
\text { Energy Index }(\text { EIn })=\text { Residual Energy / EReq(k,n) }
$$


In the above equation Residual Energy is the energy remained in the node and EReq(k,n) is the Energy required to send $\mathrm{k}$ bits to the node $\mathrm{n}$. The Energy Index in generally depends on two factors: distance to the target and the energy remained in the node. Message overhearing occurs when a node sends message. If a node is sending an message with range $d$, then all other nodes with lower distance than $d$ will hear the message. This concept can be applied for clustering.

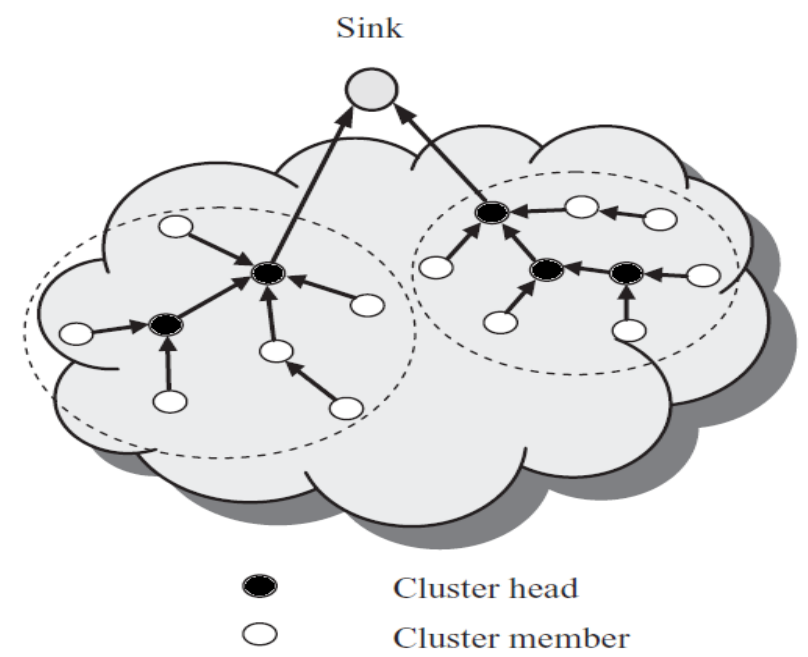

Fig. 1 Multihop Hierarchical Clustering

The major problem with clustering is how to select the cluster heads and how to organize the clusters. In this context, there are many clustering strategies. According to the distance between the cluster members and their cluster heads, a sensor network can be organized into a multihop clustering architecture, as shown in Fig.1.

The nodes are not accessible in Wireless Sensor Network, as they are energy limited. But Sink is completely accessible as it has no limitations. So we shall assume that Sink declared its location by establishing place advertisement messages. According to this policy, sink is assumed to be a mobile and it has many advantages. In mobile network, the distance between sink and node and the energy required to send data is not constant. Hence by using suitable mobile sink, the lifetime of network will considerably increase. The advantage with sink is it provides higher security for any kind of network.

Sink is the place where all the sensed information will get gathered. Hence sink is considered as an information centre. The proposed algorithm has two phases.

\subsection{Clustering Phase}

In this phase each node generates a random number between 0 and 1 and if the generated number is less than $T(n)$, then the node will be a cluster head. These cluster head nodes send messages to other nodes present in the network. The message contains cluster head identification number, and its distance to the sink. Non-head node message contains received information and distance in a table. Cluster head node save the messages from other cluster heads which are nearer to the limitation factor and then select its nearest cluster head. After performing the above actions, cluster heads will perform TDMA scheduling.

Every non-head node after receiving message will analyse the schedule and nearest hop node. The nearest hop node is a cluster head and it gathers information from all nodes.

\subsection{Transmission State}

In this phase, the non-head nodes monitor the environment and they wake up at a special time to send data together with Energy Index to the cluster head using transmitter based code assignment. All other cluster nodes will aggregate the messages received from other nodes and saves it.

\section{PERFormance ANALYSiS}

In this section, the performance of our E2MHR is compared with LEACH. We use the network simulator NS-2 to simulate the proposed algorithm. In our simulation, we use a network where 100 nodes were randomly distributed in a $100 \mathrm{~m} \times 100 \mathrm{~m}$ area, with a fixed BS located in the edge of the sensing field. The transmission range of a sensor node is $10 \mathrm{~m}$, and all sensor nodes periodically sense events and transmit the data packet to the BS. The initial energy of each sensor node is $2 \mathrm{~J}$, and the BS has unlimited energy. each data message is 512 bytes long, and the packet header for each type of packet is 25 bytes long. The 
communication energy parameter-electronics energy is set as: Eelec $=50 \mathrm{~nJ} / \mathrm{bit}$, and the energy for data aggregation is set as: Eaggr $=5 \mathrm{~nJ} / \mathrm{bit}$. For convenience, we show a part of parameters in Table 1 .

For compare our E2MHR with LEACH, we consider nodes homogenous with identical initial energy and sink assumed to be fixed.

Table 1. Simulation Parameters

\begin{tabular}{|c|c|}
\hline Parameter & Value \\
\hline Network size & $100 \mathrm{~m} \times 100 \mathrm{~m}$ \\
\hline Number of sensors & 100 \\
\hline Node initial energy $\left[E r_{i}(0)\right]$ & $2 \mathrm{~J}$ \\
\hline Transmitter circuitry dissipation $\left(E_{\text {elec }}\right)$ & $50 \mathrm{~nJ} / \mathrm{bit}$ \\
\hline Data Aggregation Energy $E_{\text {aggr }}$ & $5 \mathrm{~nJ} / \mathrm{bit}$ \\
\hline Data packet size & 512 bytes \\
\hline Packet header size & 25 bytes \\
\hline
\end{tabular}

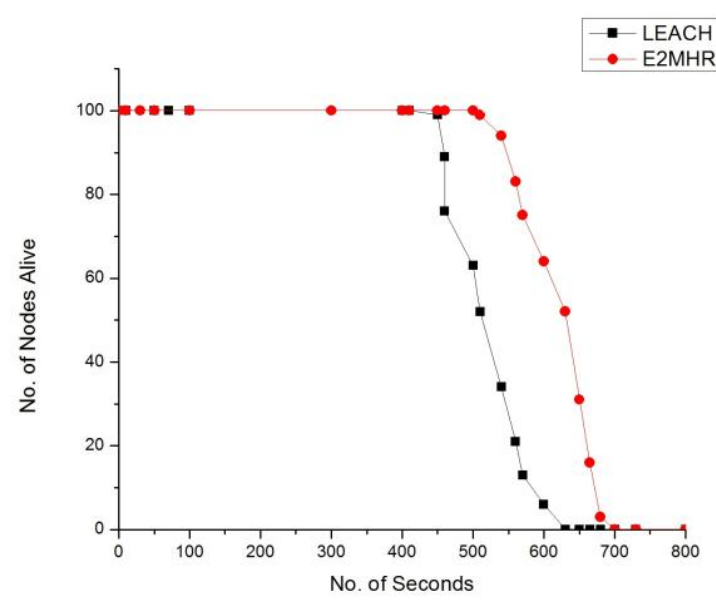

Fig. 2 Comparison Of Network Lifetime

In Fig. 2, LEACH simulation results on this network shows that first nodes die at second 409 and network stops working at 634 and our E2MHR simulation results shows that the first node dies at second 504 and network stops working at 687 .

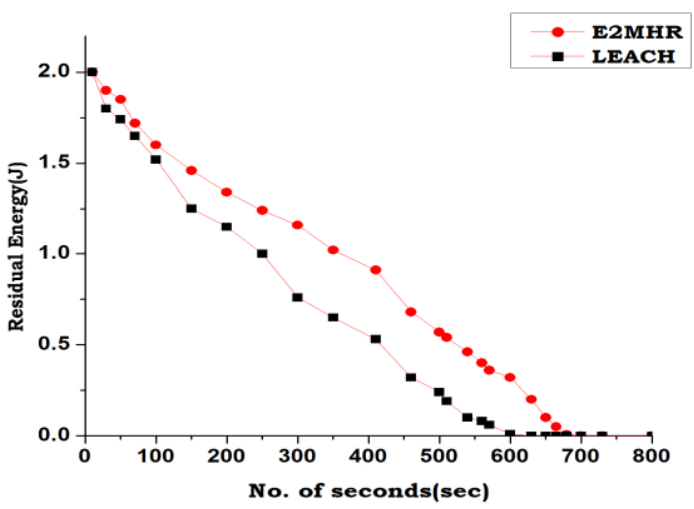

Fig. 3 Residual Energy Vs Time

By using E2MHR the lifetime of network increased, it is because of multi-hop routing and using Energy Index of the nodes.

\section{CONCLUSION}

In this proposed system, we use multi-hop hierarchical routing protocol in Wireless Sensor Network. It reduces power consumption and increases lifetime of network to reach the scalable routing. Our proposed scheme makes use of distributed algorithm and random numbers. It uses place advertisement messages of sink and other new factors for selecting the candidate node to send cluster messages. From the simulation result it is 
clear that our E2MHR has increased the lifetime of network.

\section{REFERENCES}

[1] Akyildiz.I, W. Su, Y. Sankarasubramaniam, E. Cayirci, A survey on sensor networks", IEEE Communications Magazine), 2002, 102-114

[2] Akkaya. K , M. Younis , A survey on routing protocols for wireless sensor networks", Ad-hoc Networks, Elsevier. 2003

[3] Chandrasekaran.V, Shanmugam.A, 2012 "A review on hierarchical cluster based routing in Wireless sensor networks" Journal of Global Research in Computer Science, vol. 3, no. 2,2012 pp. 12-16.

[4] Heinzelman W.B. et al., "Energy Efficient Communication Protocol for Wireless Sensor Networks", Hawaii,International Conference on System Science, Volume 2. 2000

[5] Heinzelman W. B. et al., "An Application-Specific Protocol Architecture for Wireless Microsensor Networks", IEEE, Volume 1. 2002.

[6] Younis O. and Fahmy S., "HEED: A Hybrid, Energy- Efficient, Distributed Clustering Approach for Ad Hoc Sensor Networks", IEEE Transactions on Mobile Computing, vol. 3, no. 4, 2004 , pp. 660-669.

[7] Ramanathan R. and Hain R.. 2000 Topology Control of Multihop Wireless Networks Using Transmit Power Adjustment. InProceedings Infocom 2000

[8] Rappaport T., 1996 "Wireless Communications: Principles \& Practice", Englewood Cliffs, NJ: Prentice-Hall.

[9] Wei, C., Zhi, C., Fan, P. \& Ben Letaief, K. ,2009. Asor: An energy efficient multi-hop opportunistic routing protocol forwireless sensor networks over Rayleigh fading channels, IEEE Transactions on Wireless Communications 8(5): 2452-2463.

[10] Tavli, B. \& Heinzelman, W. Mh-trace: Multihop time reservation using adaptive control for energy efficiency, IEEE Journal on Selected Areas in Communications 22(5),2004, 942-953 